East African Medical Journal Vol. 80 No. 2 February 2003

MAXILLARY INCISOR ROOT FORMS IN ORTHODONTIC PATIENTS IN NAIROBI, KENYA

P M. Ng'ang'a, BDS, MSD, Ph.D., Senior Lecturer, Department of Paediatric Dentistry and Orthodontics, Faculty of Dental Sciences, University of Nairobi, P.O. Box 19676, Nairobi, Kenya and R.N. Ng'ang'a, BDS, Lecturer, Department of Community Oral Health, Kenya Medical Training College, P.O. Box 30195, Nairobi, Kenya

Request for reprints to: Dr. P. M. Ng'ang'a, Department of Paediatric Dentistry and Orthodontics, Faculty of Dental Sciences, University of Nairobi, P.O. Box 19676, Nairobi, Kenya

\title{
MAXILLARY INCISOR ROOT FORMS IN ORTHODONTIC PATIENTS IN NAIROBI, KENYA
}

\author{
P. M. NG'ANG'A and R. N. NG'ANG'A
}

\begin{abstract}
Objective: To evaluate, radiographically, the root forms of maxillary incisors in a sample of patients seeking orthodontic treatment in Nairobi, Kenya.

Design: A retrospective study of maxillary incisor root forms based on periapical radiographs.

Setting: A private dental clinic in Nairobi, Kenya.

Materials and Methods: The study comprised 393 maxillary incisors in 100 consecutive subjects(51 boys, 49 girls) aged 9-24 years. Intra-oral periapical radiographs of the incisors were evaluated. An index was used to categorise the roots as follows: $0=$ normal, 1=short, 2-blunt, 3=apical bend, 4=pippete apex.

Results: Normal root form was recorded in $60 \%$, short in $12.5 \%$, blunt in $7 \%$, apical bend in $13 \%$, and pippete-apex in $7 \%$ of the roots. Sixty nine percent of the pippeteapex roots were in central incisors and $94 \%$ of the roots with apical bend were in lateral incisors. Seventy three percent of short roots were in females.

Conclusion: Based on evidence from previous studies on risk of root resorption which indicates that pipette and blunt roots are the forms mostly involved in this phenomenon, the present data imply that about $14 \%$ of the roots may have been at risk of moderate to severe resorption during fixed orthodontic treatment.
\end{abstract}

\section{INTRODUCTION}

Some of the well documented adverse effects usually associated with orthodontic treatment are gingival hyperplasia, marginal bone loss, enamel demineralization, pulp damage, mandibular dysfunction and root resorption. Histological and radiographic observations show that root resorption incident to orthodontic treatment occurs apically(1-3). Usually, the resorption is minor and is considered of no importance. In a few patients, however, the teeth may exhibit severe resorption. There does not seem to be one specific reason in the literature which has been implicated in severe root resorption. Rather, it appears more likely that a number of factors taken together may account for the resorption which takes place. Some of the risk factors associated with resorption include deviating root form(4-6), treatment requiring torque and the use of uprighting springs(7), prolonged treatment with CL II elastics or rectangular arches(8), severely traumatised teeth with signs of root resorption prior to orthodontic treatment $(9,10)$ and intrusive forces(11). Iatrogenic problems are becoming increasingly common issues from a medico-legal point of view. In regard to root resorption, it appears that no practitioner can be completely immune to this problem. Hence any predictor which would help warn the clinician of a likelihood of resorption in a particular patient and therefore help to prevent or reduce the risk would be of great advantage. One such predictor is root form. However, no report on the root forms of teeth are available for Kenyans. The purpose of this study was to evaluate, radiographically, the root forms of maxillary incisors in a sample of patients seeking orthodontic treatment in Nairobi, Kenya.

\section{MATERIALS AND METHODS}

This study comprised 393 maxillary incisors in 100 consecutive subjects seeking orthodontic treatment at a private clinic in Nairobi. There were 51 boys and 49 girls aged 9-24 years. Routine intra-oral periapical radiographs of incisors taken prior to orthodontic treatment were evaluated. The radiographs had been taken using a Siemens cone model number 53.37.480K 134B, Brazil. All X-rays were of good quality.

Teeth with a history of severe trauma and in which the roots were either dilacerated or obviously already resorbed, teeth previously orthodontically treated or teeth with incomplete root formation were excluded from the study. A magnifying glass $\left(\mathrm{x}^{1} / 2\right.$ ) was used to view the roots under an X-ray viewer with adjustable light intensity. Evaluation included the four maxillary incisors. The assessment of root forms followed the method described by Levander and Malmgren(6). Normal 
root form(Figure 1) was given a score of O. Deviating root forms were recorded as follows: short $=1$, blunt 1 , apical bend $=3$ and apical-pipette $=4$ (Figure 2). Only Kenyans of African descent were included in the study.

Figure 1

Normal root form in a maxillary lateral and central incisor

Figure 2

Classification of deviating root form(Levander and Malmgren(6)

\begin{tabular}{|l|}
\hline \\
\hline
\end{tabular}

1=short; 2=blunt; $3=$ apical bend; 4=apical pipette

\section{RESULTS}

Table 1 shows the distribution of the subjects according to age. Most patients were undertaking orthodontic treatment between the ages of 10-14 years.

Table 2 shows the distribution of 393 maxillary incisor roots according to root form. There were seven incisors, (4 centrals and 3 laterals) all in males, missing due to either previous trauma or impaction. Most $\operatorname{roots}(60.3 \%)$ had normal form. Blunt and apical pipette root forms comprised $6.9 \%$ and $7.4 \%$ respectively.

Table 2

Distribution of maxillary incisor roots according to root form $(n=393)$

\begin{tabular}{lcc}
\hline Apical root form & No. & $\%$ \\
\hline Normal & 237 & 60.3 \\
Short & 49 & 12.5 \\
Blunt & 27 & 6.9 \\
Apical bend & 51 & 13 \\
Apical-pippete & 29 & 7.4 \\
\hline Total & 393 & 100 \\
\hline
\end{tabular}

The distribution of 393 maxillary incisor roots according to form and tooth type (Table 3).

Table 3

Distribution of maxillary incisor roots according to form and tooth type $(n=393)$

\begin{tabular}{lcccc}
\hline $\begin{array}{l}\text { Apical root } \\
\text { form }\end{array}$ & \multicolumn{2}{c}{ Central } & incisor & \multicolumn{2}{c}{ Lateral incisor } \\
& No. & $\%$ & No. & $\%$ \\
\hline Normal & 129 & 33.7 & 108 & 27 \\
Short & 34 & 69 & 15 & \\
Blunt & 10 & 37 & 17 & 63 \\
Apical bend & 3 & 6 & 48 & 94 \\
Apical-pippete & 20 & 69 & 9 & 31 \\
\hline Total & 196 & 49.9 & 197 & 50.1 \\
\hline
\end{tabular}

There were more central incisors with short and apical-pipette roots than lateral incisors. On the other hand, there were more lateral incisors with blunt and apical bend roots than central incisors. Table 4 shows the distribution of 393 maxillary incisors according to root form and gender. There was a relatively higher percentage of short, blunt and apical-pipette root forms in females than males.

\section{Table 1}

Distribution of the subjects according to age $(M=51, F=49)$

\begin{tabular}{|c|c|c|c|c|c|c|c|c|c|c|c|c|c|c|c|c|}
\hline Age(yrs) & 9 & 10 & 11 & 12 & 13 & 14 & 15 & 16 & 17 & 18 & 19 & 20 & 21 & 22 & 23 & 24 \\
\hline No.of Children & 9 & 13 & 11 & 14 & 14 & 11 & 8 & 8 & 2 & 2 & 2 & 1 & 2 & - & 1 & 2 \\
\hline
\end{tabular}


Table 4

Distribution of 393 Maxillary incisors according to gender $(M=51, F=49)$

\begin{tabular}{|c|c|c|c|c|}
\hline \multirow{2}{*}{$\begin{array}{l}\text { Apical root } \\
\text { form }\end{array}$} & \multicolumn{2}{|c|}{ Male } & \multicolumn{2}{|c|}{ Female } \\
\hline & No. & $\%$ & No. & $\%$ \\
\hline Normal & 130 & 54.9 & 107 & 45.1 \\
\hline Short & 13 & 27 & 36 & 73 \\
\hline Blunt & 13 & 48 & 14 & 52 \\
\hline Apical bend & 27 & 53 & 24 & 47 \\
\hline Apical pipette & 13 & 45 & 16 & 55 \\
\hline Total & 196 & 49.9 & 197 & 50.1 \\
\hline
\end{tabular}

\section{DISCUSSION}

The outcome of orthodontic treatment may be jeopardised by severe apical root resorption. If there is no apical root resorption seen in the maxillary or mandibular central incisors, then significant apical resorption occurring in other teeth is less likely because the anterior teeth are the most frequently affected(7,1214). In terms of severity, the most frequently affected teeth are the maxillary lateral and central incisors. A study of the root forms of these teeth, therefore, was considered appropriate.

This was a descriptive retrospective study using intra-oral radiographs of incisors. The radiographs are standard requirement when carrying out treatment with fixed orthodontic appliances. The radiographs were therefore primarily ordered for treatment purposes and were taken at a government approved X-ray centre while observing standard radiation protection measures. Since the radiographs had been taken at the beginning of treatment, the recommended ways(15) of managing patients with the different root patterns were observed during the course of treatment. The patients confidentiality was ensured by not reproducing their personal details during data collection. In a study of root forms among the Caucasians(6), normal root forms were recorded in $63.4 \%$, short in $9.2 \%$, blunt in $12.1 \%$, apical bend in $13 \%$, and apical pipette in $2.3 \%$ of the sample. These findings are similar to the data found in the Kenyan population in this study (Table 2), except in the occurrence of pipette-shaped root forms which was relatively higher among the Kenyans.

The incisor root form most susceptible to severe resorption is the pipette shaped(6). The biconcave form means that the apical part is thin and even minor resorption would have a great effect on root length. Blunt roots have a moderate risk of root resorption. The aetiology of this root form might be a disturbance during the development of the root or an earlier superficial resorption caused by trauma(8) or non physiological forces such as nail biting acting on the tooth(16). The typical resorption of teeth with apical bends is reported to involve the bent part of the root. Short roots do not appear to exhibit more resorption than normal roots(6).

It is recommended that teeth assessed to be at moderate risk of resorption at the beginning or in the early stages of orthodontic treatment should be checked radiographically every six months during treatment, whereas teeth at high risk should be checked every three months(15). If these guidelines were to be applied in the roots in the present study, then three monthly radiographs would have been indicated in $7.4 \%$ of the sample(apical pipette root forms). About $20 \%$ would have required a six monthly review, (blunt and apical bend root forms).

Studies indicate that orthodontically induced root resorption does not usually progress after appliance removal, and histological investigations show repair of resorption sites after treatment(17). However, there is a risk of tooth mobility in a maxillary incisor that undergoes severe root resorption during orthodontic treatment if the remaining root length is equal to or less than $9 \mathrm{~mm}(18)$. It is claimed that extensive root resorption does not affect the functional capacity of a tooth. Little information is available on the long term prognosis of teeth with markedly short roots(18), but this situation must be considered important because periodontal disease could shorten the attachment apparatus further. If, therefore, severe root resorption is observed during treatment, the goals of treatment must be reassessed and a decision made to accept a treatment compromise or terminate treatment altogether(17).

Among the Caucasians, the degree of root resorption in teeth with blunt or pipette shaped roots has been shown to be significantly higher than in teeth with normal root form(15). In the present study, the percentage of teeth with pipette and blunt root form were higher in girls than boys (Table 4). The frequency of severe root resorption has been shown to be higher among girls than boys $(6,12,19,20)$. If one was to assume that there are no racial differences in the susceptibility to root resorption, the data in the present study could then imply that $14 \%$ of the roots in this sample may have been at risk of moderate to severe resorption during fixed orthodontic treatment. Obviously, further research would be needed to confirm this assumption.

\section{CONCLUSION}

The data reported here differ somewhat from those reported for Caucasians particularly with regard to the percentage of pipette shaped roots. This type of root form was observed more frequently amongst the Africans. This may be a racial difference which needs to be corroborated by further research. The present sample needs to be followed closely to find out if any root resorption occurs during the course of orthodontic treatment. 


\section{REFERENCES}

1. Reitan, K. Initial tissue behaviour during apical root resorption. Angle Orthod. 1974; 44:68-82.

2. Rygh, P. Orthodontic root resorption studied by electron microscopy. Angle Orthod. 1977; 47:1-16.

3. Harry, M.R. and Sims, M.R. Root resorption in bicuspid intrusion. Angle Orthod. 1982; 52:235-258.

4. Oppenheim, A. Human tissue response to orthodontic intervention of short and long duration. Am. J. Orthod. Oral. Surg. 1942; 28:26-33.

5. Newman, W.G. Possible etiologic factors in external root resorption. Am. J. Orthod. 1975; 67:522-539.

6. Levander, E. and Malmgren, O. Evaluation of the risk of root resorption during orthodontic treatment. A study of upper incisors. Eur. J. Orthod. 1988; 10:30-38.

7. Goldson, L. and Henrikson, C.O. Root resorption during Begg treatment: A longitudinal roentgenologic study. Am. J. Orthod. 1975; 68:55-66.

8. Linge, B.O. and Linge, L. Apical root resorption in upper anterior teeth. Eur. J. Orthod. 1983; 5:173-183.

9. Malmgren, O., Goldson, L., Hill, C., Orwin, A., Petrini, L. and Lundberg, M. Root resorption after orthodontic treatment of traumatized teeth. Am. J. Orthod. 1982; 82:487-491.

10. Hines, F.B., Jr. A radiographic evaluation of the response of previously avulsed teeth and partially avulsed teeth to orthodontic movement. Am. J. Orthod. 1979; 75:1-19.
11. Dermaut, L.R. and De Munck, A. Apical root resorption of upper incisors caused by intrusive tooth movement: A radiographic study. Am. J. Orthod. 1986; 90:321-326.

12. Massler, M. and Perreault, L.G. Root resorption in permanent teeth of young adults, J. Dent. ChId. 1954; 21:158-164.

13. De Shields, R.W. A study of root resorption in treated Class II division I malocclusions. Angle. Orthod. 1969; 39:231245.

14. Sjolien, T. and Zachrisson, B. Periodontal bone support and tooth length in orthodontically treated and untreated persons. Am. J. Orthod. 1973; 64:28-37.

15. Levander, E., Bajka, R. and Malmgren, O. Early radiographic diagnosis of apical root resorption during orthodontic treatment: A study of maxillary incisors. Eur. J. Orthod. 1998; 16:223-229.

16. Odenrick, L. and Brattsrom, V. The effect of nail-biting on root resorption during orthodontic treatment. Eur. J. Orthod. 1983; 5:185-188.

17. Copeland, S. and Green, L.J. Root resorption in maxillary central incisors following active orthodontic treatment. Am. J. Orthod. 1986; 89:51-55.

18. Levander, E. and Malmgren, O. Long-term follow-up of maxillary incisors with severe apical root resorption. Eur. J. Orthod. 2000; 22:85-92.

19. Hopkins, R. and Adams, D. Multiple idiopathic resorption of teeth. Brit. Dent. J. 1979; 146:309-312.

20. George, D.I. and Miller, R.L. Idiopathic resorption of teeth. Am. J. Orthod. 1986;89:13-20. 